\title{
Conversion of Furfuryl Alcohol into Ethyl Levulinate over Glucose-Derived Carbon-Based Solid Acid in Ethanol
}

\author{
Geng Zhao ${ }^{1, *}$, Ming Liu ${ }^{1}$, Xinkui Xia ${ }^{2}, \mathrm{Li} \mathrm{Li}^{1}$ and Bayin $\mathrm{Xu}^{1}$ \\ 1 Analysis and Testing Center, College of Chemistry and Chemical Engineering, Xinyang Normal University, \\ Xinyang 464000, Henan, China; lmminga@163.com (M.L.); xytclily@163.com (L.L.); \\ burenbayin@163.com (B.X.) \\ 2 College of Food Science, Xinyang Agriculture and Forestry University, Xinyang 464000, Henan, China; \\ xynzxb@126.com \\ * Correspondence: zhaogeng@xynu.edu.cn; Tel.: +86-376-639-3155
}

Received: 27 March 2019; Accepted: 15 May 2019; Published: 16 May 2019

\begin{abstract}
In this study, a carbon-based solid acid was created through the sulfonation of carbon obtained from the hydrothermal pretreatment of glucose. Additionally, ethyl levulinate, a viable liquid biofuel, was produced from furfuryl alcohol using the environmentally benign and low-cost catalyst in ethanol. Studies for optimizing the reaction conditions, such as reaction time, temperature, and catalyst loading, were performed. Under the optimal conditions, a maximum ethyl levulinate yield of $67.1 \%$ was obtained. The recovered catalyst activity (Ethyl levulinate yield 57.3\%) remained high after being used four times, and it was easily regenerated with a simple sulfonation process. Moreover, the catalyst was characterized using FT-IR, XRD, SEM, elemental analysis, and acid-base titration techniques.
\end{abstract}

Keywords: furfuryl alcohol; ethanol; ethyl levulinate; carbon-based solid acid

\section{Introduction}

Because of the recent diminishment of fossil fuel resources, as well as environmental degradation resulting from greenhouse gas emissions, significant effort has been devoted to converting renewable biomass into liquid fuels, fuel additives, and organic bulk chemicals [1-3]. Ethyl levulinate (EL) is considered to be a potential liquid biofuel for the future [4]. Ethyl levulinate is a short chain fatty ester, with properties similar to the fatty acid methyl ester of biodiesel. It has many excellent benefits, such as high lubricity, non-toxicity, flashpoint stability, and good flow properties under cold conditions [5]. Moreover, EL is of particular interest due to its extensive applications in the flavoring, solvent, and plasticizer sectors [6]. Additionally, it has found applications in the area of organic chemistry for the synthesis of the viable biofuel $\gamma$-valerolactone $[7,8]$.

Currently, acid-catalyzed esterification of levulinic acid (LA) is the most efficient method for the synthesis of EL, with high yields regularly achieved [9-12]. However, LA is an expensive raw material, because it needs to be prepared from carbohydrates or biomass firstly, and then needs to be purified for synthesis of ethyl levulinate $[13,14]$. On the other hand, an increasing number of studies have focused on the direct production of EL from biomass in ethanol, such as hexose [15-17], cellulose [18], wood, bagasse [19], and wheat straw [20]. As a direct feedstock, raw biomass is abundant and inexpensive; however, the highest yield (55\%) was achieved starting from the aforementioned feedstock (hexose).

As can be seen from Scheme 1, furfuryl alcohol (FA) is produced industrially via the hydrogenation of furfural $[21,22]$. It should be noted that furfural can be derived from the hydrolysis and dehydration of xylan contained in hemicellulose-rich biomass, including corncobs, corn stock, rice hulls, and olive 
stones. Presently, there are more than 400 factories producing furfural in China, and the annual global production of furfural exceeds 700,000 tons [23,24]. Therefore, furfural and FA are not fully utilized in the chemical market [25]. The conversion of FA into EL is regarded as an economic and convenient strategy that has several advantages, including lower reaction temperature, cheaper raw material, and higher product yield. However, little effort has been made to employ this approach to produce EL. Some researchers have obtained EL from FA catalyzed by sulfuric acid [24], acidic ion-exchange resins [26], sulfonic acid functionalized ILs [27], and aluminosilicates [28]. It is important to note that these catalysts, except for sulfuric acid, can be extremely expensive due to their complex preparation processes. Furthermore, sulfuric acid and sulfonic acid functionalized ILs are homogeneous, creating serious drawbacks in terms of separation, recycling, and equipment corrosion. Therefore, it is necessary to develop an environmentally benign and low-cost solid acid catalyst for the conversion of FA into EL. In the resulting studies, high EL yields were achieved, but under longer reaction time $(24 \mathrm{~h})$ or lower substrate concentration (1.0 wt.\%), which may potentially be hurdles in the process of the commercialization of EL.

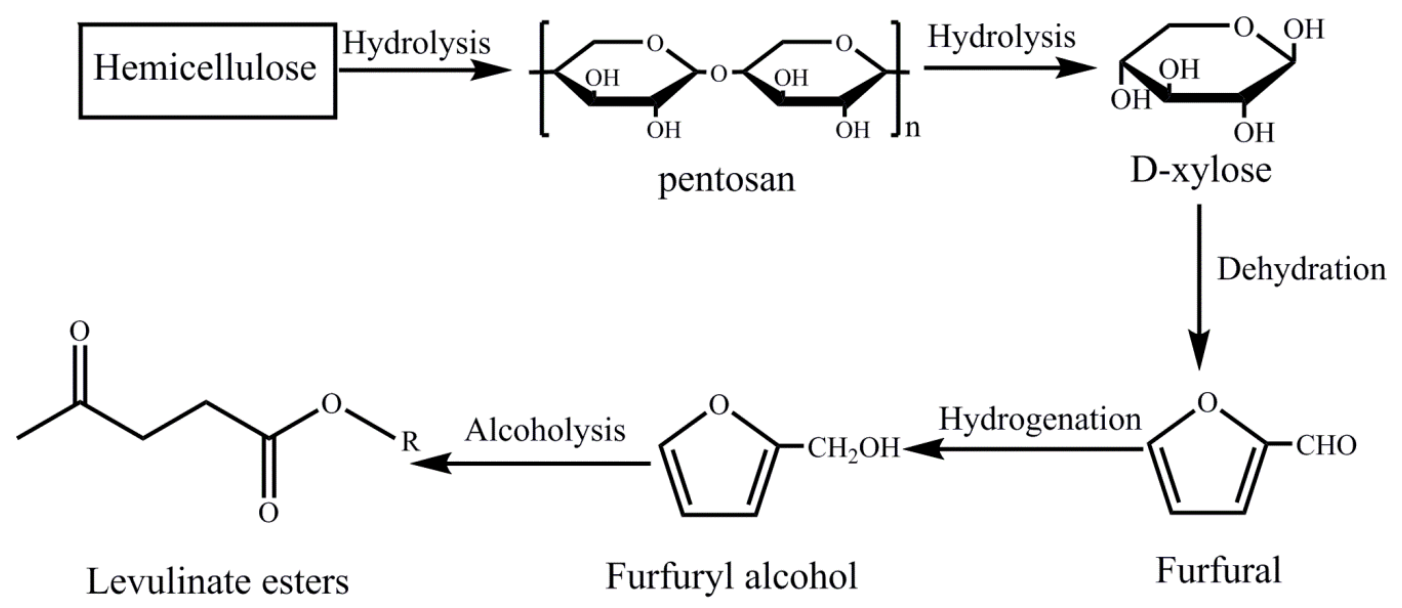

Scheme 1. The route of synthesizing levulinate esters from hemicellulose.

In this study, an inexpensive and robust carbon-based solid acid catalyst was successfully synthesized and evaluated for the conversion of FA into EL in ethanol. A maximum EL yield of $67.1 \%$ was achieved under the optimal conditions, and all the conversion reactions were performed in triplicate. The recovered catalyst possessed good catalytic activity (EL yield 57.3\%), even after four cycles, and it was easily regenerated by a simple sulfonation process. Moreover, the catalyst was characterized using FT-IR, XRD, SEM, elemental analysis, and acid-base titration techniques.

\section{Results and Discussion}

\subsection{Catalyst Characterizations}

Structural information about unsulfonated glucose-derived carbon (UGC) and glucose-derived carbonaceous catalyst (GCC) samples were obtained by FT-IR and XRD. Figure 1 shows the XRD patterns of two samples, which exhibit two $2 \theta^{\circ}$ weak diffraction peaks between 10 and $30^{\circ}$ and 35 and $50^{\circ}$, which can be assigned to $C(002)$ and $C(101)$, respectively. The results indicate that amorphous carbon, composed of aromatic carbon sheets, is oriented in a random fashion [29]. Two samples have similar XRD patterns, indicating that the sulfonation process had no effect on the morphology of carbon.

The UGC and GCC were further characterized by FT-IR, and the results are shown in Figure 2. The vibration bands at 1384,1172 , and $1035 \mathrm{~cm}^{-1}$ ( $\mathrm{SO}_{3}$ stretching) on GCC are evidence that $-\mathrm{SO}_{3} \mathrm{H}$ groups were successfully incorporated into the UGC. In addition, the absorption bands at 1715 and $1620 \mathrm{~cm}^{-1}$ correspond to $-\mathrm{C}=\mathrm{O}$ (carbonyl) and $-\mathrm{OH}$ (hydroxyl) bending vibrations, implying that carboxyl groups exist on the prepared carbon materials. The bands at 3424 and $1620 \mathrm{~cm}^{-1}$ can be 
assigned to $\mathrm{C}-\mathrm{OH}$ stretching and $-\mathrm{OH}$ bending vibrations, implying that hydroxyl groups exist on the prepared carbon materials [30,31].

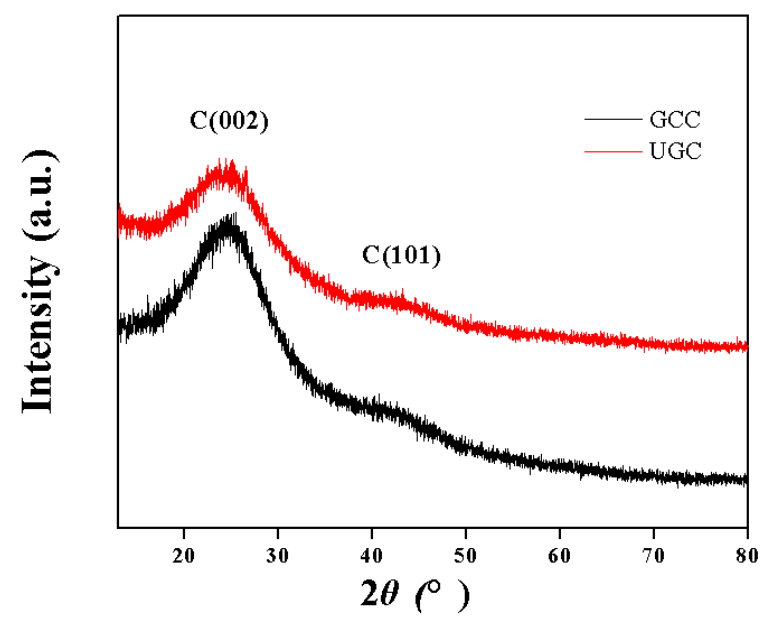

Figure 1. XRD patterns of unsulfonated glucose-derived carbon (UGC) and glucose-derived carbonaceous catalyst (GCC).

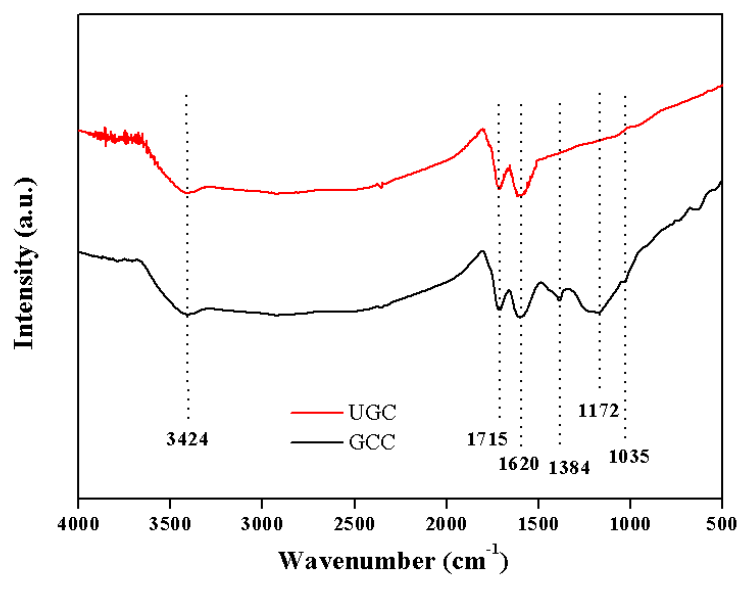

Figure 2. FT-IR spectra of UGC and GCC.

\subsection{Identification of the Product}

The mechanism responsible for the conversion of FA to EL is complex. Additionally, it is also possible for FA to react with ethanol at higher temperatures to form ether, poly FA, and other by-products [24,27]. In an attempt to identify the product, the liquid phase of the reaction mixture obtained from the conversion of FA in the presence of GCC (at $423 \mathrm{~K}$ for $60 \mathrm{~min}$ ) was analyzed using GC-MS. The GC-MS spectra are given in Figures S1 and S2, located in the Supplementary Materials section. GC-MS analysis of the reaction mixture shows the presence of EL at a retention time of $10.54 \mathrm{~min}$. The mass spectrum of the product exhibits molecular ionization at $\mathrm{m} / \mathrm{z} 144$, corresponding to the molecular formula of EL $\left(\mathrm{C}_{7} \mathrm{H}_{12} \mathrm{O}_{3}\right)$. Furthermore, the base peak fragmentation of the ester is related to $\mathrm{CH}_{3} \mathrm{CO}(\mathrm{m} / \mathrm{z}=43)$. Two other important ion peaks, located at $\mathrm{m} / \mathrm{z} 99$ and 129 , are due to the formation of the ion acylium $\mathrm{RCO}^{+}$, resulting from the loss of either the alkoxy or methyl group from the ester. Additional bond cleavages occurred through some pathways and created fragment ions at $40,55,74$, and $101 \mathrm{~m} / \mathrm{z}$ [10]. 


\subsection{Effect of Reaction Parameters}

The catalytic conversions of FA were conducted with temperatures ranging from 373 to $423 \mathrm{~K}$, using differing time intervals, to deduce the optimum conditions for increasing the EL yield. As seen in Figure 3, the reaction temperature and time have a great influence on the overall EL yield. Under lower temperature condition, only $35.5 \%$ EL yield was achieved after the reaction time of $150 \mathrm{~min}$ at $373 \mathrm{~K}$. Elevating the reaction temperature to $423 \mathrm{~K}$ increased the yield of EL rapidly with the extension of the reaction time, and the equilibrium point $(67.1 \%)$ was reached by $60 \mathrm{~min}$. Moreover, at 398 and $423 \mathrm{~K}$, when EL yields reached their peak values, longer reaction times resulted in lower yields of EL. The low yield of EL indicates that the substance might decompose to some extent, and more undesired byproducts were formed under higher temperatures [32].

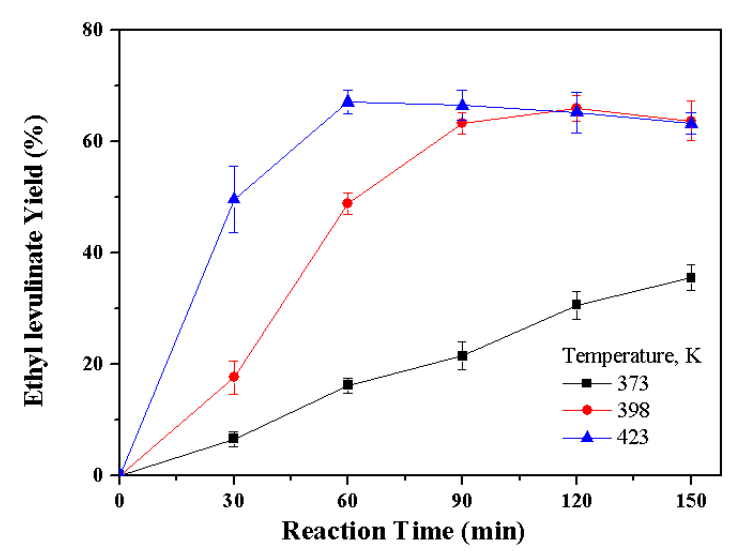

Figure 3. Effect of reaction temperature on EL yield. Reaction conditions: furfuryl alcohol (FA) $1 \mathrm{~g}$, ethanol $19 \mathrm{~g}$, GCC $0.5 \mathrm{~g}$.

Subsequently, the effect of the catalyst loading dosage on the conversion of FA into EL was studied, and the results are presented below in Figure 4. When the reaction time was $60 \mathrm{~min}$ and $1 \mathrm{wt} . \%$ GCC was used, the EL yield was only $26.5 \%$. Increasing the GCC dosage to $2.5 \mathrm{wt} . \%$ resulted in a remarkable increase in EL yield to $67.1 \%$. However, when the catalyst loading dosage was doubled to $5.0 \mathrm{wt} . \%$, the yield of EL increased by only $1.3 \%$. Moreover, after $60 \mathrm{~min}$, the EL yield decreased faster when $5.0 \mathrm{wt} . \%$ GCC was used, which could be attributed to the increased availability of acid sites, derived from excessive GCC, facilitating the decomposition of EL. Thus, it was determined that a $2.5 \mathrm{wt} . \%$ catalyst loading dosage was optimal.

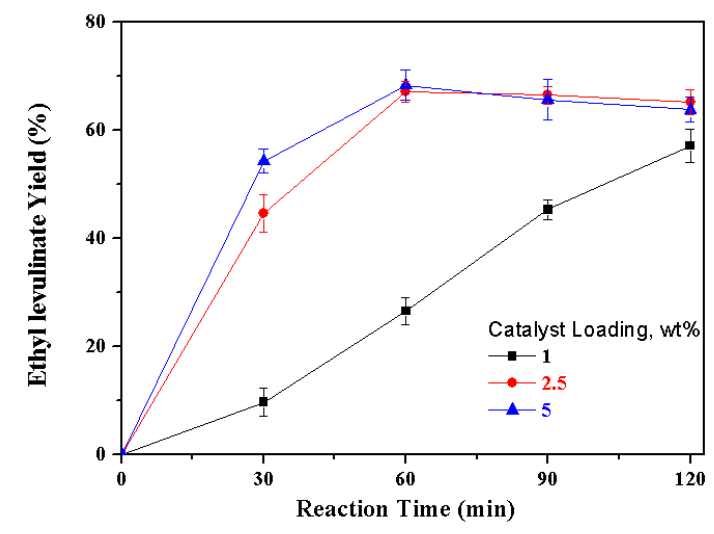

Figure 4. Effect of catalyst dosage on EL yield. Reaction conditions: FA $1 \mathrm{~g}$, ethanol $19 \mathrm{~g}$, temperature 423K. 


\subsection{Recyclability of GCC}

The reusability and long-term stability of a given catalyst are extremely important factors in reducing production cost for practical biomass transformation. After the reaction was completed, the GCC was separated from the reaction mixture by filtration, washed 10 times with $10 \mathrm{~mL}$ of ethanol, and dried at $378 \mathrm{~K}$ for $24 \mathrm{~h}$ in a vacuum oven. Then, the GCC was used in the next reaction experiment under the same reaction conditions. As shown in Figure 5, GCC maintained good catalytic activity after four reactions, and an EL yield of $57.3 \%$ was still achieved. Subsequently, the four time-recycled GCC was regenerated by simple sulfonation at $453 \mathrm{~K}$ for $1 \mathrm{~h}$ using a solid/concentrated sulfuric acid ratio of 1:10 under a nitrogen atmosphere. The yield of the regenerated EL was restored to $66.4 \%$, which was comparable to that of the fresh catalyst (67.1\%).

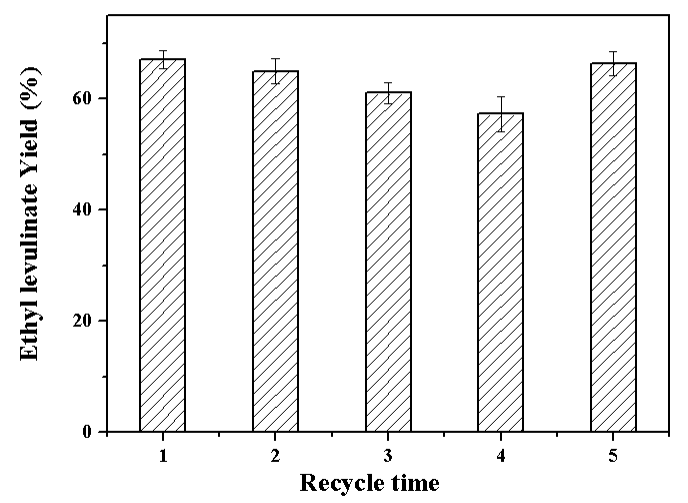

Figure 5. Reuse of the GCC catalyst. Reaction conditions: FA $1 \mathrm{~g}$, ethanol $19 \mathrm{~g}$, GCC $0.5 \mathrm{~g}$ temperature $423 \mathrm{~K}$, reaction time $60 \mathrm{~min}$.

Under the same experimental conditions (at $423 \mathrm{~K}$ for $60 \mathrm{~min}$ ), the catalytic activity of UGC (EL yield 11.6\%) is much lower than that of GCC (EL yield 67.1\%). The remarkable catalytic activity of GCC may be due to the high acidity of the sulfonated carbon catalyst, as well as the presence of $-\mathrm{SO}_{3} \mathrm{H}$ groups on the amorphous carbon catalyst. The acid density and elemental content of the reclaimed GCC are summarized in Table 1. The acid density and the sulfuric content of GCC decreased significantly with the increasing reaction cycles. On the other hand, the carbon content of GCC increased from 52.2 to $60.6 \mathrm{wt} . \%$ throughout the course of the four reaction runs. The results indicated that the slight decrease in EL yield was possible due to the leaching of the $-\mathrm{SO}_{3} \mathrm{H}$ group coupled with the deposition of humins on the surface of the GCC studied. In addition, the acid density and elemental content of the regenerated GCC were nearly identical to the fresh catalyst, indicating that the regeneration process not only recovered the catalytic activity of GCC, but also utilized the humins deposited on GCC.

Table 1. Total acid density and elemental content of the reclaimed GCC.

\begin{tabular}{cccc}
\hline \multirow{2}{*}{ Run } & \multirow{2}{*}{ Total Acid Density $\left(\mathbf{m m o l} \cdot \mathbf{g}^{\mathbf{- 1}}\right)^{\mathbf{a}}$} & \multicolumn{2}{c}{ Element Content $(\mathbf{w t .} \%)^{\mathbf{b}}$} \\
\cline { 3 - 4 } & & Carbon & Sulfur \\
\hline 1 & 2.6 & 52.2 & 1.5 \\
2 & 1.9 & 57.9 & 1.1 \\
3 & 1.7 & 59.2 & 1.0 \\
4 & 1.4 & 60.6 & 0.9 \\
$5^{\mathrm{c}}$ & 2.5 & 53.1 & 1.5 \\
\hline
\end{tabular}

${ }^{a}$ Determined by acid-base titration. ${ }^{\mathrm{b}}$ Obtained by elemental analysis. ${ }^{\mathrm{c}}$ The regenerated GCC.

The comparative SEM images of the fresh and four time-recycled GCC are shown in Figure 6. It is clear that they both exhibited an intertwined structure and consisted of irregular particles with a size of several micrometers. However, the particles of the four time-recycled GCC were larger, and their 
surfaces were smoother, indicating substantial humin deposition on the surface of GCC after four reaction runs.

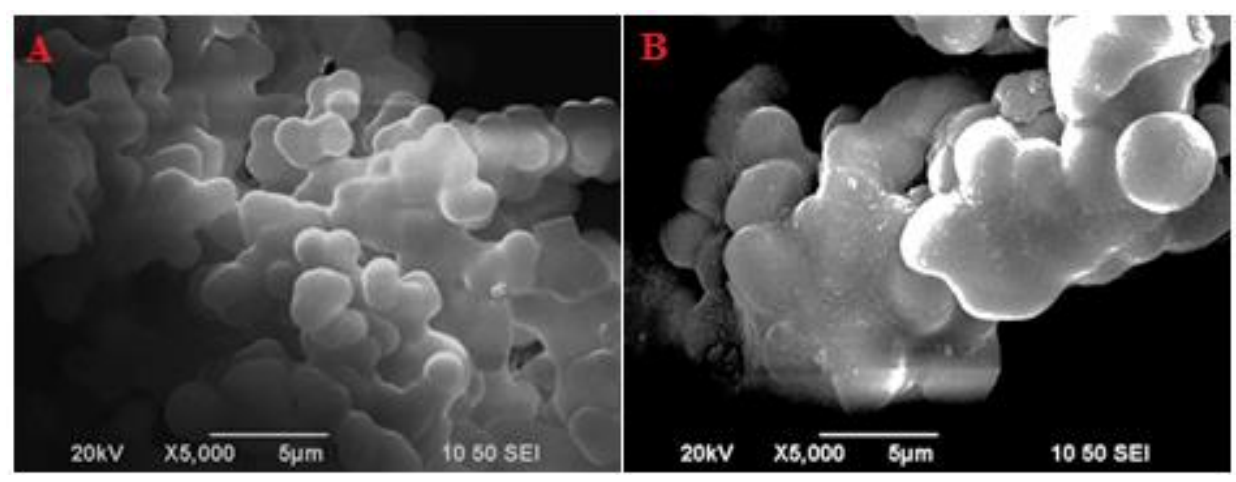

Figure 6. SEM images for fresh catalyst (A) and recycled catalyst after four runs (B).

\section{Materials and Methods}

\subsection{Materials}

Ethyl levulinate and furfuryl alcohol were purchased from Aladdin Chemical Reagent (Shanghai, China). Other reagents were obtained from Sinopharm Chemical Reagent (Shanghai, China). All reagents and chemicals were analytical grade and used without further purification or treatment.

\subsection{Catalyst Preparation}

Using the typical preparation method, glucose $(30 \mathrm{~g})$ was dissolved in $50 \mathrm{~mL}$ of distilled water in a cylindrical stainless steel pressurized reactor with $100 \mathrm{~mL}$ total volume (PARR, Moline, IL, USA). The reactor was sealed and heated to $473 \mathrm{~K}$ with agitation rate of $500 \mathrm{rpm}$ for $6 \mathrm{~h}$. After the hydrothermal carbonization process was completed, the solid carbon was separated by filtration, washed with distilled water, and subsequently dried in an oven at $383 \mathrm{~K}$ for $24 \mathrm{~h}$. The resulting solid carbon $(5 \mathrm{~g})$ was then ground to a powder and heated at $453 \mathrm{~K}$ for $5 \mathrm{~h}$ in $50 \mathrm{~mL}$ of concentrated sulfuric acid $(98 \%)$ under a nitrogen-rich atmosphere to introduce $-\mathrm{SO}_{3} \mathrm{H}$ groups. The sulfonated mixture was then diluted with $1000 \mathrm{~mL}$ of deionized water to form a black precipitate, which was subsequently washed repeatedly in hot distilled water $(>353 \mathrm{~K})$ until the sulfate ions were no longer detected in the residual washing water. Finally, the resulting glucose-derived carbonaceous catalyst (GCC) was dried in an oven at $383 \mathrm{~K}$ for $24 \mathrm{~h}$.

\subsection{Catalyst Characterization}

The prepared GCC was characterized by Fourier transform infrared spectroscopy (FT-IR), X-ray diffraction spectroscopy (XRD), scanning electron microscopy (SEM), elemental analysis, and acid-base titration. The FT-IR spectrum was recorded over the range of 400 to $4000 \mathrm{~cm}^{-1}$ on a Nicolet iS 50 FT-IR spectrometer, made by Thermo Fisher Scientific, Waltham, MA, USA. The XRD pattern of the GCC was recorded by an X' Pert PRO powder XRD spectrometer (Panalytical, Almelo, The Netherlands) using a $\mathrm{Cu} \mathrm{K} \alpha$ radiation source $(\lambda=0.154 \mathrm{~nm})$. The morphology of the GCC was observed using a S-4800 scanning electron microscope (Hitachi, Tokyo, Japan) at $30 \mathrm{kV}$. The elemental content of the GCC sample was determined using a Vario EL III elemental analyzer, made by Elementar, Hanau, Germany. The acid density of the GCC was determined via acid-base titration.

\subsection{General Procedure for the Synthesis of EL}

The synthesis of EL from FA were conducted in a 50-mL cylindrical, stainless steel, pressurized reactor (PARR, Moline, IL, USA). The reactor was heated in an adjustable electric stove. In a typical experiment, FA $(1 \mathrm{~g})$, ethanol $(19 \mathrm{~g})$, and a given amount of GCC were introduced into the reactor. 
After the reactor was sealed, the above mixture was heated to the desired temperature and stirred magnetically at $500 \mathrm{rpm}$. At the end of the reaction, the resulting mixture was cooled to room temperature. The liquid products and solid acid catalyst were separated through centrifugation at $10,000 \mathrm{rpm}$ for $3 \mathrm{~min}$. After the process was completed, the contents were analyzed.

\subsection{Analysis of Products}

The qualitative analysis of the sample was conducted with a Shimadzu 2010A gas chromatograph (GC) system, coupled with a Shimadzu QP2010 mass spectrometer (GC-MS). The amount of product was analyzed by GC on an Agilent 7890 instrument, equipped with a HP-5 capillary column and a flame ionization detector (FID). The injection port temperature was $523 \mathrm{~K}$, and the detector temperature was $543 \mathrm{~K}$. The column temperature was maintained at $313 \mathrm{~K}$ for $4 \mathrm{~min}$, then raised to $523 \mathrm{~K}$ with a ramp rate of $15 \mathrm{~K} / \mathrm{min}$. The amount of EL present at the end of the process was calculated using an external standard. The yield of EL on a molar basis was calculated as follows:

$$
\text { EL yield }(\%)=\frac{E L(\mathrm{~mol})}{\mathrm{FA}(\mathrm{mol})} \times 100 \%
$$

\section{Conclusions}

An environmentally benign and inexpensive solid acid catalyst GCC was demonstrated to be highly active for use in the conversion of FA into EL in ethanol. A good EL yield of 67.1\% was obtained under moderate temperature and short reaction times (423 K, $60 \mathrm{~min})$. GCC could be recycled four times, with little deactivation, and could be easily regenerated with a simple sulfonation process.

Supplementary Materials: The following are available online. Figure S1: GC spectra of the product of the conversion of FA into EL., Figure S2: MS spectra of the product of the conversion of FA into EL.

Author Contributions: G.Z. conceived and designed the experiments; M.L. and X.X. performed the experiments; L.L. analyzed the data; B.X. contributed reagents and analysis tools; and G.Z. wrote the paper.

Funding: This work was financially supported by the National Natural Science Foundation of China (21606188), the Foundation of Henan Educational Committee (15A530011), the Nanhu Scholars Program for Young Scholars of XYNU, and the Doctoral Research Foundation of Xinyang Normal University.

Acknowledgments: This research was supported by the Analysis \& Testing Center of Xinyang Normal University. We would like to thank Liu Peifang and Zhang Zongwen for the assistant and technique support in SEM.

Conflicts of Interest: The authors declare no conflict of interest.

\section{References}

1. Bozell, J.J. Chemistry connecting biomass and petroleum processing with a chemical bridge. Science 2010, 329, 522-523. [CrossRef]

2. Ullah, K.; Sharma, V.K.; Dhingra, S.; Braccio, G.; Ahmad, M.; Sofia, S. Assessing the lignocellulosic biomass resources potential in developing countries: A critical review. Renew. Sustain. Energy Rev. 2015, 51, 682-698. [CrossRef]

3. Climent, M.J.; Corma, A.; Iborra, S. Conversion of biomass platform molecules into fuel additives and liquid hydrocarbon fuels. Green Chem. 2014, 16, 516-547. [CrossRef]

4. Joshi, H.; Moser, B.R.; Toler, J.; Smith, W.F.; Walker, T. Ethyl levulinate: A potential bio-based diluent for biodiesel which improves cold flow properties. Biomass Bioenerg. 2011, 35, 3262-3266. [CrossRef]

5. Zhao, S.; Xu, G.; Chang, C.; Fang, S.; Liu, Z.; Du, F. Direct conversion of carbohydrates into ethyl levulinate with potassium phosphotungstate as an efficient catalyst. Catalysts 2015, 5, 1897-1910. [CrossRef]

6. Hayes, D.J. An examination of biorefining processes, catalysis and challenges. Catal. Today 2009, 145, 138-151. [CrossRef]

7. Tang, X.; Chen, H.; Hu, L.; Hao, W.; Sun, Y.; Zeng, X.; Lin, L.; Liu, S. Conversion of biomass to $\gamma$-valerolactone by catalytic transfer hydrogenation of ethyl levulinate over metal hydroxides. Appl. Catal. B 2013, 147, 827-834. [CrossRef] 
8. Peng, L.; Tao, R.; Wu, Y. Catalytic upgrading of biomass-derived furfuryl alcohol to butyl levulinate biofuel over common metal salts. Catalysts 2016, 6, 143. [CrossRef]

9. Fernandes, D.R.; Rocha, A.S.; Mai, E.F.; Mota, C.J.A.; Teixeira da Silva, V. Levulinic acid esterification with ethanol to ethyl levulinate production over solid acid catalysts. Appl. Catal. A 2012, 425-426, 199-204. [CrossRef]

10. Lee, A.; Chaibakhsh, N.; Rahman, M.B.A.; Basri, M.; Tejo, B.A. Optimized enzymatic synthesis of levulinate ester in solvent-free system. Ind. Crops Prod. 2010, 32, 246-251. [CrossRef]

11. Pasquale, G.; Vázquez, P.; Romanelli, G.; Baronetti, G. Catalytic upgrading of levulinic acid to ethyl levulinate using reusable silica-included Wells-Dawson heteropolyacid as catalyst. Catal. Commun. 2012, 18, 115-120. [CrossRef]

12. Oliveira, B.L.; Teixeira da Silva, V. Sulfonated carbon nanotubes as catalysts for the conversion of levulinic acid into ethyl levulinate. Catal. Today 2014, 234, 257-263. [CrossRef]

13. Cha, J.; Hanna, M.A. Levulinic acid production based on extrusion and pressurized batch reaction. Ind. Crops Prod. 2001, 16, 109-118. [CrossRef]

14. Rackemann, D.W.; Doherty, W.O.S. The conversion of lignocellulosics to levulinic acid. Biofuels Bioprod. Bioref. 2011, 5, 198-214. [CrossRef]

15. Peng, L.; Lin, L.; Zhang, J.; Shi, J.; Liu, S. Solid acid catalyzed glucose conversion to ethyl levulinate. Appl. Catal. A 2011, 397, 259-265. [CrossRef]

16. Peng, L.; Lin, L.; Li, H. Extremely low sulfuric acid catalyst system for synthesis of methyl levulinate from glucose. Ind. Crops Prod. 2012, 40, 136-144. [CrossRef]

17. Saravanamurugan, S.; Van Buu, O.N.; Riisager, A. Conversion of mono- and disaccharides to ethyl levulinate and ethyl pyranoside with sulfonic acid functionalized ionic liquids. ChemSusChem 2011, 4, 723-726. [CrossRef]

18. Garves, K. Acid catalyzed degradation of cellulose in alcohols. J. Wood Chem. Technol. 1988, 8, 121-134. [CrossRef]

19. Mao, R.L.V.; Zhao, Q.; Dima, G.; Petraccone, D. New process for the acid catalyzed conversion of cellulosic biomass (AC3B) into alkyl levulinates and other esters using a unique one-pot system of reaction and product extraction. Catal. Lett. 2011, 141, 271-276. [CrossRef]

20. Chang, C.; Xu, G.; Jiang, X. Production of ethyl levulinate by direct conversion of wheat straw in ethanol media. Bioresour. Technol. 2012, 121, 93-99. [CrossRef]

21. Merlo, A.B.; Vetere, V.; Ruggera, J.F.; Casella, M.L. Bimetallic PtSn catalyst for the selective hydrogenation of furfural to furfuryl alcohol in liquid-phase. Catal. Commun. 2009, 10, 1665-1669. [CrossRef]

22. Nagaraja, B.M.; Padmasri, A.H.; Raju, B.D.; Rao, K.S.R. Vapor phase selective hydrogenation of furfural to furfuryl alcohol over Cu-MgO coprecipitated catalysts. J. Mol. Catal. A 2007, 265, 90-97. [CrossRef]

23. Sanchez, C.; Serrano, L.; Andres, M.A.; Labidi, J. Furfural production from corn cobs autohydrolysis liquors by microwave. Ind. Crops Prod. 2013, 42, 513-519. [CrossRef]

24. Maldonado, G.M.G.; Assary, R.S.; Dumesic, J.A.; Curtiss, L.A. Acid-catalyzed conversion of furfuryl alcohol to ethyl levulinate in liquid ethanol. Energy Environ. Sci. 2012, 5, 8990-8997. [CrossRef]

25. Wang, G.; Zhang, Z.; Song, L. Efficient and selective alcoholysis of furfuryl alcohol to alkyl levulinates catalyzed by double SO3H-functionalized ionic liquids. Green Chem. 2014, 16, 1436-1443. [CrossRef]

26. Lange, J.P.; Van de Graaf, W.D.; Haan, R.J. Conversion of furfuryl alcohol into ethyl levulinate using solid acid catalysts. ChemSusChem 2009, 2, 437-441. [CrossRef] [PubMed]

27. Hengne, A.M.; Kamble, S.B.; Rode, C.V. Single pot conversion of furfuryl alcohol to levulinic esters and $\gamma$-valerolactone in the presence of sulfonic acid functionalized ILS and metal catalysts. Green Chem. 2013, 15, 2540-2547. [CrossRef]

28. Neves, P.; Lima, S.; Pillinger, M.; Rocha, S.M.; Rocha, J.; Valente, A.A. Conversion of furfuryl alcohol to ethyl levulinate using porous aluminosilicate acid catalysts. Catal. Today 2013, 218, 76-84. [CrossRef]

29. Nakajima, K.; Hara, M. Environmentally benign production of chemicals and energy using a carbon-based strong solid acid. J. Am. Ceram. Soc. 2007, 90, 3725-3734. [CrossRef]

30. Chen, G.; Fang, B. Preparation of solid acid catalyst from glucose to starch mixture for biodiesel production. Bioresour. Technol. 2011, 102, 2635-2640. [CrossRef] 
31. Yang, J.; Li, G.; Zhang, L.; Zhang, S. Efficient Production of N-Butyl Levulinate Fuel Additive from Levulinic Acid Using Amorphous Carbon Enriched with Oxygenated Groups. Catalysts 2018, 8, 14. [CrossRef]

32. Hronec, M.; Fulajtarova, K.; Sotak, T. Kinetics of high temperature conversion of furfuryl alcohol in water. J. Ind. Eng. Chem. 2014, 20, 650-655. [CrossRef]

Sample Availability: Not available.

(C) 2019 by the authors. Licensee MDPI, Basel, Switzerland. This article is an open access article distributed under the terms and conditions of the Creative Commons Attribution (CC BY) license (http://creativecommons.org/licenses/by/4.0/). 